\title{
OBITUARIO
}

\section{In Memoriam doctora Augusta Noemí Ochoa López \\ (1937-2015)}

\section{In Memoriam doctor Augusta Noemí Ochoa López \\ (1937-2015)}

\section{Gabriela Rouillon ${ }^{1}$, Maribel Baylon ${ }^{2}$ y Silvia Aguilar ${ }^{2}$}

1 Colaboradora independiente

2 Facultad de Ciencias Biológicas, Universidad Nacional Mayor de San Marcos. Apartado Postal 110058, Lima 11, Perú.

Email Gabriela Rouillon: gabriela.rouillon@euskodat.net

Email Maribel baylon: mbaylonc@unmsm.edu.pe

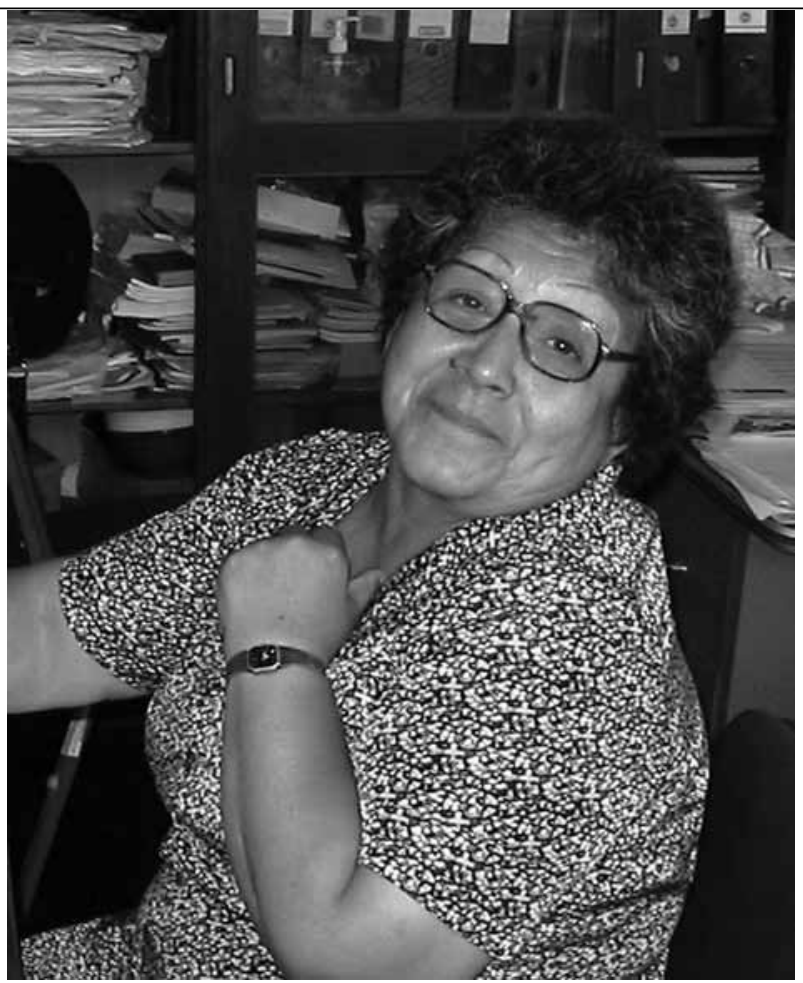

Doctor Augusta Noemí Ochoa López

Solo la dedicación silenciosa que tuviste para con tus pasiones, te llevó a ser lo que fuiste en cada uno de tus rincones, aquellos que todos conocimos y aquellos otros que reservaste celosamente para los que te amaron.

La Doctora Augusta Noemí Ochoa López, nació un martes 4 de mayo de 1937 en Cañete, Lima. Empezó los estudios de la carrera de biología en la Universidad Nacional Mayor de San Marcos, en 1953, también llevo cursos de la carrera de Educación. Obtuvo su grado de Bachiller en Biología

\section{Citación:}

Rouillon G., M. Baylon \& S. Aguilar. 2015. In Memoriam doctora Augusta

Noemí Ochoa López (1937-2015). Revista peruana de biología 22(2)

135 - 138 (Agosto 2015). doi: http://dx.doi.org/10.15381/rpb.v22i2.11349 


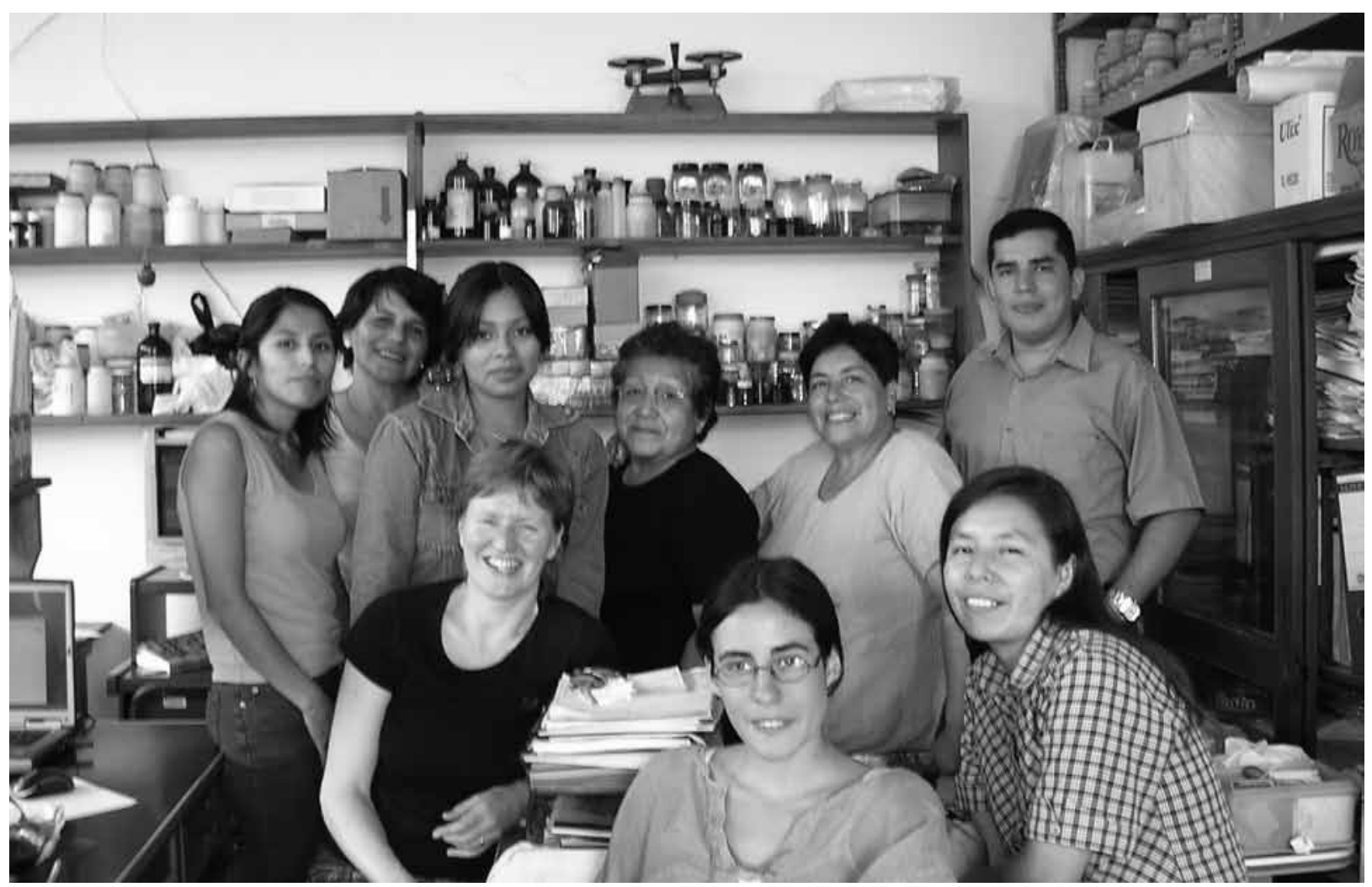

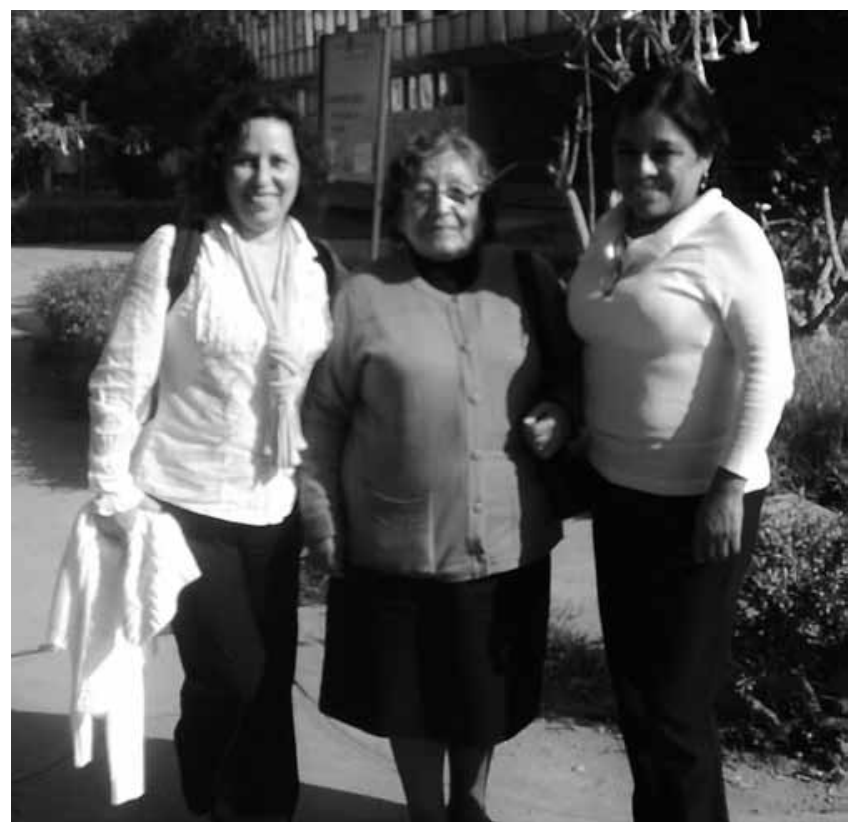

Doctora Noemí Ochoa con Gabriela Rouillon y Sivia Aguilar (2013-05-27)

en el año 1966 con la Tesis "Observaciones sobre el Desove de la Anchoveta, Engraulis ringens J., en la zona del Callao". Este trabajo lo desarrolló en el Instituto del Mar del Perú (IMARPE), que a su vez se convirtió en su primer centro de trabajo y el que le permitió formarse como investigadora. Aquí, en el IMARPE, se integró al grupo de trabajo sobre plancton, donde comenzó a forjar su pasión por el fitoplancton.

El fitoplancton como tema de investigación es muy importante, considerando que es el principal alimento de la anchoveta (Engraulis ringens), una de las pesquerías más importantes del mundo y un recurso económico para el país; además, el mar peruano caracterizado por sus intensos afloramientos lo convierten en uno de los ecosistemas de más alta productividad. Noemi centró su trabajo sobre aspectos taxonómicos del fitoplancton, que involucraban la identificación de organismos muy diversos siendo pionera en esta área de los estudios marinos en el Perú. Además de las investigaciones, en el IMARPE, como personal altamente calificado, también participaba en reuniones internacionales sobre los temas de plancton. Noemí llegó a ocupar la Jefatura de Plancton, que con los años pasó a llamarse el Laboratorio de Fitoplancton y Productividad Primaria como parte de la Dirección de Oceanografía y Cambio Climático.

En 1988, obtuvo su grado de Doctor en Ciencias con la Tesis: "Dinoflagelados del mar peruano y su valor como indicadores de masas de agua”. Unos años después, en 1991 ingresó a la docencia universitaria en la Facultad de Ciencias Biológicas de Universidad Nacional Mayor de San Marcos, como profesor Principal de los cursos de Oceanografía General y Plancton Marino y también como colaboradora del curso de Hidrobiología General. Organizó e impartió varios cursos internacionales de fitoplancton y zooplancton: oceánico, antártico. Como docente continuó con sus investigaciones participando en diferentes proyectos de investigación en la universidad, siempre con el fitoplancton como tema.

Noemí estuvo más de 50 años dedicada con verdadera pasión al estudio del fitoplancton marino, dedicación silenciosa y una disciplina inimitable fueron las características de su trabajo. Fue una docente ejemplar, que siempre apoyo a sus alumnos, aunque sus temas de investigación no fueran los mismos que el de ella, era pues una persona para la que la amistad primaba. Varias promociones de alumnos la hicieron madrina, en reconocimiento a su calidad de docente y de persona. 


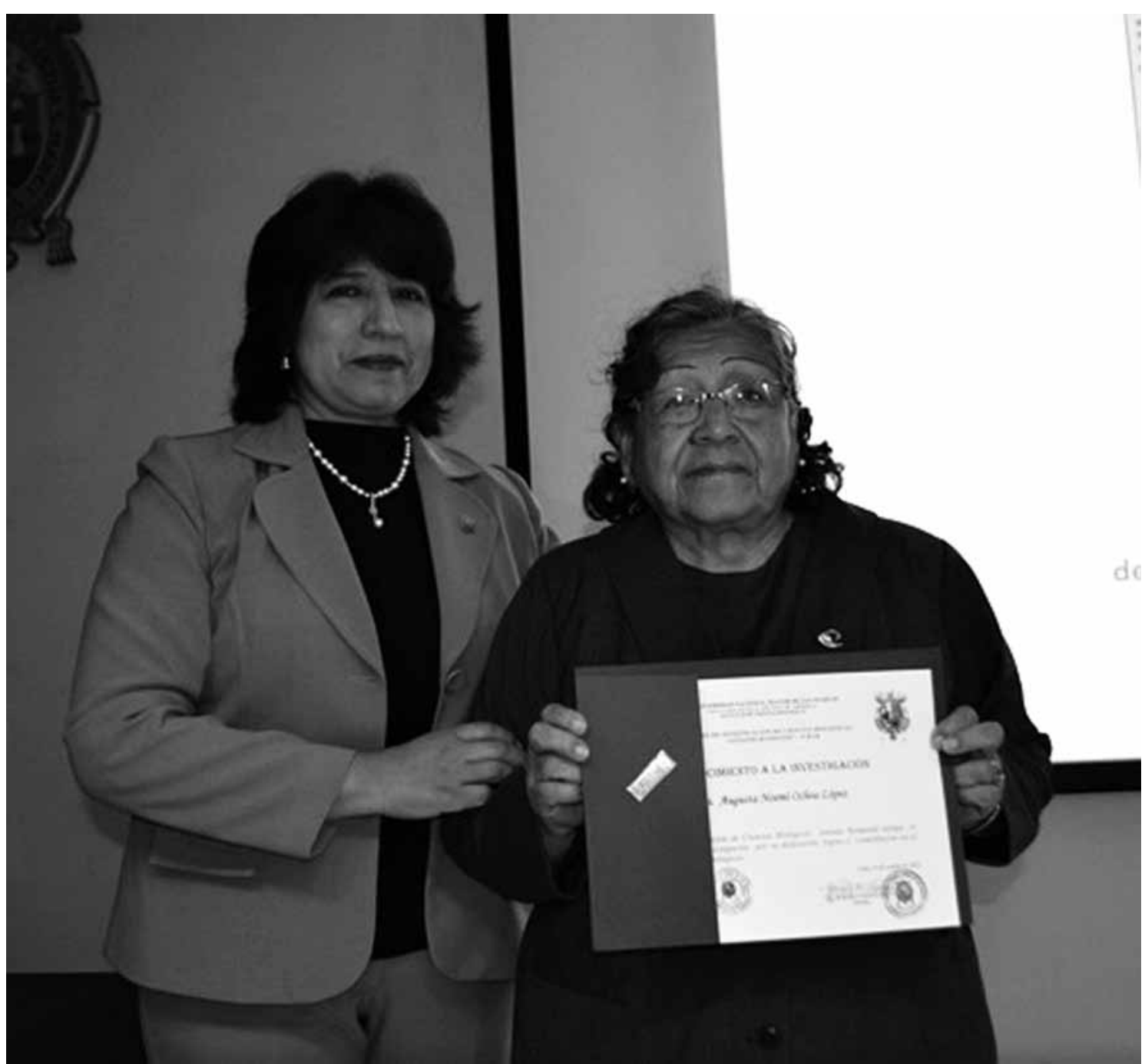

Doctora Noemí Ochoa con Decana Martha valdivia en reconocimiento a su labor (octubre 2012)

Es así que, en el año 2012 Noemí Ochoa cesó en su labor docente y posteriormente en julio de 2014, en reconocimiento de su labor académica fue nombrada Profesora Emérita de la Universidad Nacional Mayor de San Marcos, ambos fueron momentos muy emotivos para ella y los que las rodeabamos, uno porque significaban un alejamiento a su constante actividad de investigadora y de su más recientemente labor docente y otro por que la comunidad académica de la universidad le reconocia su labor.

La Dra. Noemí partió de entre nosotros el 2 de mayo de 2015, dejó una huella profunda en aquellos que la rodearon, tanto por su calidad como persona, como por su labor de investigación y docencia. Recordaremos de ella su constancia, paciencia, cordura, honestidad y franqueza, pero también la recordaremos como la científica infatigable, apasionada por su tema de investigación, exigente en el conocimiento y en la disciplina.

\section{Principales publicaciones científicas de la doctora Noemí Ochoa}

[1] N. Ochoa, «Observaciones sobre el desove de la anchoveta, Engraulis ringens J., en la zona del Callao», Tesis de Bachiller, Universidad Nacional Mayor de San Marcos, Lima, Perú, 1966.

[2]B. Rojas De Mendiola, N. Ochoa, R. Calienes, y O. Gómez, «Contenido estomacal de anchoveta en cuatro áreas de la costa peruana», Informe Instituto del Mar del Perú, vol. 27, pp. 1 - 29, 1969.
[3]B. Rojas De Mendiola y N. Ochoa, «Observations on the food and feeding habits of the anchovy Engraulis ringens Jenyns made during cruise 6908-09", en Oceanography of the south Pacific 1972, R. Fraser, Ed. Wellington, New Zealand, 1973, pp. 457-461.

[4]B. Rojas De Mendiola y N. Ochoa, «Phytoplankton and spawning of anchoveta», en IOC Workshop Report No. 28, Paris: IOC/UNESCO, 1980.

[5]W. G. Harrison, R. Platt, R. Calienes, y N. Ochoa, «Photosynthetic parameters and primary production of phytoplankton population off the Northern Coast of Perú», en Coastal Upwelling, F. . Richards, Ed. Washington D. C.: American Geophysical Union, 1981, pp. 323-349.

[6] N. Ochoa y O. Gómez, «Variaciones del fitoplancton en el área de Chimbote durante 1977.», In: A. Landa (ed): Investigación Cooperativa de la Anchoveta y su Ecosistema (ICANE) entre Perú y Canadá. Boletín Instituto del Mar del Perú, vol. extraordinario, pp. 119-129, 1981.

[7] N. Ochoa, Metodología usada en el Instituto del Mar del Perú para la colección y análisis de muestras de fitoplancton, vol. Informe sobre Ciencias del Mar No 11. UNESCO, 1981.

[8]B. Rojas De Mendiola, N. Ochoa, y O. Gómez, «Los dinoflagelados como indicadores biológicos de masas de agua-fenómeno El Niño 1972.», en Memorias del Seminario sobre Indicadores Biolócos del Plancton, Montivedeo, Uruguay: Oficina regional de la Ciencia y Tecnología de la UNESCO para América Latina y del Caribe, 1981, pp. 78-88.

[9]P. Hendrikson, K. G. Sellner, B. R. De Mendiola, N. Ochoa, y R. Zimmermann, «The composition of particulate organic matter and biomass in the Peruvian upwelling region during ICANE 1977 (Nov. 14 - Dec. 2)», Journal of Plankton Research, vol. 4, n. ${ }^{\circ}$ 1, pp. 163-186, 1982. 
[10] N. Ochoa, B. Rojas De Mendiola, y O. Gómez, «Identificación del fenómeno El Niño a través de los organismos fitoplanctónicos», In: Arntz, W., Landa, A. and Tarazona, J. (eds.), «El Niño»: Su impacto en la fauna marina. Boletín - Instituto del Mar del Perú, vol. Volumen extraordinario, pp. 23-31, 1985.

[11]B. Rojas De Mendiola, O. Gómez, y N. Ochoa, «Efectos del Fenómeno "El Niño" 1982 - 1983, sobre el fitoplancton de la costa peruana», Simp. Int. Afl. O. Afr., Inst. Inv. Pesq., Barcelona, vol. 1, pp. 417-433, 1985.

[12] N. Ochoa y O. Gómez, «Dinoflagellates as indicators of water masses during El Niño, 1982-1983", J. Geophys. Res., vol. 92, n. ${ }^{\circ}$ C13, pp. 14355-14367, dic. 1987.

[13] N. Ochoa y O. Gómez, «Variación espacio temporal del fitoplancton frente al Callao, Perú, en 1986.», En: Salzwedel H. \& Landa A. ( Eds. ) . Recursos y dinámica del ecosistema del afloramiento peruano. Boletín Instituto del Mar Perú Callao, vol. Volumen extraordinario, pp. 51 - 57, 1988.

[14] N. Ochoa, «Dinoflagelados del mar peruano y su valor como indicadores de masa de agua», Tesis doctoral, Universidad Nacional Mayor de San Marcos, Lima, Perú, 1988.

[15]J. Tarazona, C. Paredes, E. Canahuire, N. Ochoa, L. Romero, B. Shiga, y J. Pino, «El Bentos y su interacción en el Ecosistema (Parte V)», Theorema - UNMSM, vol. 1, n. ${ }^{\circ}$ 1, 1995.

[16] N. Ochoa y O. Gómez, «Dinoflagelados del mar peruano como indicadores de masas de agua durante los ańos 1982 a 1985», Boletín Instituto del Mar del Perú, vol. 16, n. ${ }^{\circ}$, pp. 1 - 60, 1997.

[17] N. Ochoa, O. Gómez, S. Sanchez, y E. Delgado, «Diversidad de Diatomeas y Dinoflagelados marinos del Perú», Boletín Instituto del Mar del Perú, vol. 18, n. ${ }^{\circ} 1-2$, pp. 1-13, 1999.

[18]J. Tarazona, A. Indacochea, S. Valle, C. Córdova, N. Ochoa, W. Serrano, y T. Peńa, «Impacto de "El Niño 1997-98" sobre el ecosistema marino somero de la costa central del Perú», Rev peru biol, vol. 6, n. ${ }^{\circ} 3$, pp. 018 - 031, 1999.

[19]J. Tarazona, W. Arntz, S. Valle, y T. Peña, «Los índices de El Niño y del impacto sobre las comunidades bentónicas», en El Niño en América Latina: Impactos biológicos y sociales, J. Tarazona, W. Arntz, y E. Castillo De Maruenda, Eds. Consejo Nacional de Ciencia y Tecnología- CONCYTEC, 2001, pp. 113 - 126.

[20]N. Ochoa y G. Rouillon, "Variabilidad del fitoplancton en la Bahía de Ancón, Lima, Perú durante El Niño 1997 - 98», En Memorias El Niño en América Latina. Impactos Biológicos y Sociales, J. Tarazona, W. Arntz y E Castillo de Maruenda, Eds. Consejo Nacional de Ciencia y TecnologíaCONCYTEC, Lima, 2001, pp. $39-45$.
[21]G. Rouillon, J. Mendo, y N. Ochoa, «Fitoplancton en el contenido estomacal de Argopecten purpuratus (Mollusca, Bivalvia) suspendida a diferentes profundidades en Bahía Independencia», en Bases ecológicas y socioeconómicas para el manejo de los recursos vivos de la Reserva Nacional de Paracas, J. Mendo y M. Wolff, Eds. Lima, Perú: Universidad Nacional Agraria La Molina, 2002, pp. 60-67.

[22] N. Ochoa y J. Tarazona, "Variabilidad temporal de pequeña escala en el fitoplancton de Bahía Independencia, Pisco, Perú», Revista Peruana de Biología, vol. 10, n. ${ }^{\circ}$ 1, pp. 5966, dic. 2003.

[23]G. Rouillon, J. Guerra Rivas, N. Ochoa, y E. Navarro, «Phytoplankton composition of the stomach contents of the mussel Mytilus edulis L. from two populations: Comparison with its food supply», Journal of Shellfish Research, vol. 24, n. ${ }^{\circ}$ 1, pp. 5-14, 2005.

[24] N. Ochoa y M. Baylón, «Dinofurcula cf. ventralis en la costa central del Perú y primeros registros de dos especies de Protoperidinium», Revista Peruana de Biología, vol. 12, n. ${ }^{\circ}$ 3, pp. 377-382, oct. 2005.

[25]J. Tam, M. H. Taylor, V. Blaskovic, P. Espinoza, R. Michael Ballón, E. Díaz, C. Wosnitza-Mendo, J. Argüelles, S. Purca, P. Ayón, L. Quipuzcoa, D. Gutiérrez, E. Goya, N. Ochoa, y M. Wolff, «Trophic modeling of the Northern Humboldt Current Ecosystem, Part I: Comparing trophic linkages under La Niña and El Niño conditions», Progress in Oceanography, vol. 79, n. ${ }^{\circ}$ 2-4, pp. 352-365, 2008.

[26]M. H. Taylor, J. Tam, V. Blaskovic, P. Espinoza, R. Michael Ballón, C. Wosnitza-Mendo, J. Argüelles, E. Díaz, S. Purca, N. Ochoa, P. Ayón, E. Goya, D. Gutiérrez, L. Quipuzcoa, y M. Wolff, «Trophic modeling of the Northern Humboldt Current Ecosystem, Part II: Elucidating ecosystem dynamics from 1995 to 2004 with a focus on the impact of ENSO", Progress in Oceanography, vol. 79, n. ${ }^{\circ}$ 2-4, pp. 366-378, 2008.

[27]J. Bonicelli P., D. López P., N. Ochoa L., y R. S. Shreeve, «Estructura comunitaria del zooplancton asociada con el fitoplancton y las masas de agua del Estrecho de Bransfield y la Isla Elefante durante el verano austral del 2006», Ecología Aplicada, vol. 7, n. ${ }^{\circ} 1-2$, pp. 159-164, dic. 2008.

[28] N. Ochoa, M. H. Taylor, S. Purca, y E. Ramos, «Intra- and interannual variability of nearshore phytoplankton biovolume and community changes in the northern Humboldt Current system», Journal of Plankton Research, vol. 32, n. ${ }^{\circ} 6$, pp. $843-855,2010$ 